\title{
$\begin{array}{ll}\text { Research Square } & \text { Preprints are preliminary reports that have not undergone peer review. } \\ \text { They should not be considered conclusive, used to inform clinical practice, } \\ \text { or referenced by the media as validated information. }\end{array}$ \\ Comparison of Clinical Features of Acute Esophageal Mucosal Lesions and Those of Reflux Esophagitis Los Angeles Classification Grade D
}

Chikamasa Ichita ( $\Delta$ ichikamasa@yahoo.co.jp)

Shonan Kamakura General Hospital

\section{Akiko Sasaki}

Shonan Kamakura General Hospital

Chihiro Sumida

Shonan Kamakura General Hospital

Takashi Nishino

Shonan Kamakura General Hospital

Jun Kubota

Shonan Kamakura General Hospital

Karen Kimura

Shonan Kamakura General Hospital

Kento Shionoya

Shonan Kamakura General Hospital

Makomo Makazu

Shonan Kamakura General Hospital

Sakue Masuda

Shonan Kamakura General Hospital

Kazuya Koizumi

Shonan Kamakura General Hospital

Makoto Kako

Shonan Kamakura General Hospital

\section{Research Article}

Keywords: acute esophageal mucosal lesion, comorbidities, esophageal reflux, black esophagus, acute necrotizing esophagitis

Posted Date: January 28th, 2022

DOI: https://doi.org/10.21203/rs.3.rs-1128026/v1 
License: (c) (i) This work is licensed under a Creative Commons Attribution 4.0 International License. Read Full License 


\section{Abstract}

Background: Acute esophageal mucosal lesions (AEMLs) cause circumferential mucosal damage in the lower esophagus in patients with deteriorating general health. However, its etiology remains unclear. AEML is often diagnosed and treated as severe reflux esophagitis (SRE), particularly that of Los Angeles classification grade D (sRE-D). This study aimed to determine whether it is appropriate to classify these two diseases according to the characteristics of the proximal mucosa of erosion as previously reported, and to clarify the clinical features of AEML in comparison with SRE-D.

Methods: Patients were classified into AEML and SRE-D groups, based on previous reports. Patient background, presence of shock, medical history, medications, comorbidities, death during hospitalization, blood sampling data, endoscopic findings, and presence of stenosis after treatment were evaluated.

Results: There were significantly more cases with comorbidities in the AEML group than in the SRE-D group, particularly comorbidities involving infectious diseases. In addition, abnormalities in blood test results were significantly more frequent in the AEML group than in the SRE-D group. This suggests that the onset of AEML may be due to peripheral circulatory disturbance caused by worsening general condition.

Conclusions: AEML and SRE-D showed distinctly different features and it was considered reasonable to distinguish them. AEML, unlike sRE-D, may be caused by microcirculatory disturbances related to comorbidities rather than gastric acid reflux. However, it is under-recognized and should be considered in the setting of upper gastrointestinal bleeding in patients with deteriorating general condition.

\section{Background}

In 2006, Tsumura et al. reported acute esophageal mucosal lesions (AEMLs) in a patient with upper gastrointestinal bleeding and diffuse mucosal injury of the esophagus who underwent upper endoscopy [1]. The disease is characterized by a tendency to occur in patients with a deteriorating systemic condition [2-5], and is often diagnosed as severe reflux esophagitis (SRE) because of its lack of recognition. Black esophagus and acute necrotizing esophagitis are severe forms of AEML [2]. The details of its pathogenesis remain unknown, although various causes, such as gastric acid reflux and microcirculatory disturbances, have been suggested [2-5]. Currently, the concept of AEML is not fully recognized, and most cases are diagnosed and treated as SRE, particularly SRE that is categorized as grade D (sRE-D) according to the Los Angeles classification [6]. SRE requires long-term prescription of acid inhibitors, while AEML can be cured within a short period by treatment of coexisting diseases and by short-term use of acid-suppressive drugs. Therefore, differentiating between these diseases is important.

Sakata et al. reported that an important difference between SRE and AEML is the shape of the esophageal mucosal erosions on the proximal side [7]. sRE is characterized by the formation of spiny erosions (Figure 1), whereas these are absent in AEML (Figure 2). Sakata et al. compared reflux esophagitis of Los Angeles grades C and D with AEML. However, it is primarily necessary to differentiate 
between SRE-D and AEML in clinical practice. In this study, we aimed to verify whether it is reasonable to distinguish the two diseases according to the characteristics of the proximal mucosal damage as in the study by Sakata et al. and to clarify the characteristics of AEML in comparison with sRE-D, which is a well-known disease.

\section{Methods}

\section{Study design}

This was a single-center, retrospective study.

\section{Patients and definitions}

In this study, patients who had undergone upper endoscopy for upper gastrointestinal bleeding and presenting with features, such as hematemesis, black vomiting, and black stool, between 2016 and 2020, and who were found to show diffuse mucosal damage occupying the entire circumference of the esophageal wall, were included. We defined the SRE-D group as patients with reflux esophagitis with Los Angeles grade $D$, with sharp, spiny, pointed erosions of the mucosa on the proximal edge of the esophagus (Figure 1). The AEML group were defined as follows: those with circumferential diffuse mucosal damage extending over at least the lower third of the esophagus, with inflammation of the mucosa sharply bordered by the squamocolumnar junction (Figure 3); and with erosions on the proximal edge of the esophagus that were not sharply pointed (Figure 2). Black esophagus was also included in the AEML group, if it met these definitions. Patients who were diagnosed with corrosive esophagitis, radiation esophagitis, infectious esophagitis, or reflux esophagitis due to obstructive symptoms caused by tumors were excluded from this study based on an interview with the patient, patient history, and endoscopic findings.

We compared the following points between these two groups: age, sex, chief complaint (hematemesis, black vomit, and black stool), presence of shock vitality, medical history (hypertension, diabetes mellitus, chronic kidney disease, coronary artery disease, liver cirrhosis, and treatment of malignancy), medications (nonsteroidal anti-inflammatory drugs [NSAIDs], antithrombotic drugs, steroids, antibiotics, and acid-secretion inhibitors), comorbidities at onset, death during hospitalization, blood sampling data (white blood cell [WBC], C-reactive protein [CRP], hemoglobin, blood urea nitrogen [BUN], creatinine, total protein, albumin, blood glucose, $\mathrm{pH}$, and lactate), endoscopic findings (lesions indicated for hemostasis, esophageal hiatal hernia, gastric ulcer, duodenal ulcer, and atrophic gastritis), and presence of stricture after treatment. In the AEML group, we also evaluated the shape of the proximal mucosa.

Shock was defined as a shock index of $>1$ at the time of presentation. Blood sampling data were collected at the time of initial consultation. Acid-secretion inhibitors included proton-pump inhibitors (PPI), histamine $\mathrm{H} 2$-receptor antagonists, and potassium-competitive acid blockers. Prerenal failure was defined as the creatinine level above $1.5 \mathrm{mg} / \mathrm{dL}$ and a fractional excretion of sodium below $1 \%$. Lesions requiring hemostasis were defined as cases in which endoscopic hemostasis was performed. Esophageal 
hiatal hernia was defined as a widening of the hilum with two or more scopes in the retroflex view of the endoscope (Figure 4). Atrophic gastritis, in which a mucosal change is caused by Helicobacter pylori infection, was diagnosed by the vascular pattern associated with loss of gastric mucosal glands and loss of folds $[8,9]$. Patients in whom it was difficult to pass the upper endoscope (GIF-H260 Olympus, Tokyo, Japan) were considered to have stenosis.

\section{Statistical analysis}

Values are expressed as mean \pm standard deviation. Categorical variables were compared using the Fisher's exact test. Differences in mean values between the two groups were compared using unpaired ttests. A two-sided $p$-value of $<0.05$ was considered to denote statistical significance. All statistical analyses were performed with EZR [10], a package for R statistical software (https://www.r-project.org/). More precisely, it is a modified version of $\mathrm{R}$ commander designed to add statistical functions frequently used in biostatistics.

\section{Results}

There were 64 patients in the AEML group and 38 patients in the sRE-D group. Patient characteristics are shown in Table 1. Compared with sRE-D, AEML was more common in a younger $(p<0.01)$ age group and in male patients $(p<0.01)$. Although there were no significant differences in complaints or the presence of shock $(p=0.08)$, patients with AEML were significantly more likely to have been treated for malignancy $(p<0.05)$. There was no significant difference in the type of medication used, except for the NSAIDs $(p<0.05)$, which were significantly more common in AEML. However, less than $45 \%$ of the AEML group used acid-suppressive drugs ( $p=0.34$ ), suggesting that the occurrence of AEML may be related to factors other than gastric acid.

Table1: Patient characteristics 


\begin{tabular}{|c|c|c|c|}
\hline & AEML $(n=64)$ & sRE-D ( $n=38)$ & $P$ value \\
\hline Age (years) & $72.9 \pm 14.71$ & $81.6 \pm 12.77$ & $p=0.003$ \\
\hline range & $(34-93)$ & $(49-95)$ & \\
\hline \multirow[t]{2}{*}{ Sex } & 35 males $(54.7 \%)$ & 10 males $(26.3 \%)$ & $p=0.005$ \\
\hline & 29 females (45.3\%) & 28 females $(73.7 \%)$ & \\
\hline \multirow[t]{3}{*}{ Chief complaint } & Hematemesis: 22 (48\%) & Hematemesis:14 (36.8\%) & $p=0.77$ \\
\hline & Black vomiting:36 (30\%) & Black vomiting:19 (50\%) & \\
\hline & Black stool:6 (22\%) & Black stools:5 (13.2\%) & \\
\hline Shock at presentation & $24(37.7 \%)$ & $8(21.1 \%)$ & $p=0.08$ \\
\hline \multicolumn{4}{|l|}{ Medical history } \\
\hline 口Hypertension & $30(46.9 \%)$ & $18(47.4 \%)$ & $p=0.96$ \\
\hline 口Diabetes Mellitus & $16(25 \%)$ & $4(10.5 \%)$ & $p=0.07$ \\
\hline ¿Chronic Kidney disease & $12(18.8 \%)$ & $6(15.8 \%)$ & $p=0.70$ \\
\hline ¿Coronary artery disease & $9(14.1 \%)$ & $1(2.6 \%)$ & $p=0.06$ \\
\hline aLiver cirrhosis & $1(1.6 \%)$ & $1(2.6 \%)$ & $p=0.71$ \\
\hline $\mathrm{QTT}$ Treatment of malignancy & $18(28.1 \%)$ & $4(10.5 \%)$ & $p=0.03$ \\
\hline \multicolumn{4}{|l|}{ Medications } \\
\hline [NSAIDs & $11(17.1 \%)$ & $1(2.6 \%)$ & $p=0.02$ \\
\hline$\square$ Antithrombotics & $17(26.6 \%)$ & $16(42.1 \%)$ & $p=0.10$ \\
\hline पSteroids & $2(3.1 \%)$ & $1(2.6 \%)$ & $p=0.89$ \\
\hline$\square$ Antibiotics & $7(10.9 \%)$ & $1(2.6 \%)$ & $p=0.13$ \\
\hline$\square$ Acid inhibitors & $28(43.8 \%)$ & $13(34.2 \%)$ & $p=0.34$ \\
\hline Death during admission & $11(17.2 \%)$ & $2(5.3 \%)$ & $p=0.08$ \\
\hline Stenosis & $1(1.6 \%)$ & $0(0 \%)$ & \\
\hline
\end{tabular}

Data are given as numbers (percentages), or as mean \pm SD, unless otherwise specified.

Although there was no significant difference, death during hospitalization was relatively common in the AEML group $(p=0.08)$. As shown in Table 2 , the AEML group had significantly more comorbidities $(p<0.001)$, including infections $(p<0.05)$. As shown in Table 3, WBC $(p<0.05), C R P(p<0.001), B U N$ 
$(p<0.01)$, creatinine $(p<0.01)$, blood glucose $(p<0.05)$, and lactate $(p<0.001)$ levels were significantly higher in the AEML group, while $\mathrm{pH}(\mathrm{p}<0.01)$ was significantly lower in the $A E M L$ group.

Table 2

Comorbidities at presentation

\begin{tabular}{|llll|}
\hline & AEML $(\mathbf{n}=\mathbf{6 4})$ & SRE-D $(\mathbf{n}=\mathbf{3 8})$ & P value \\
\hline Comorbidities & $38(59.4 \%)$ & $7(18.4 \%)$ & $\mathrm{p}=0.0001$ \\
\hline$\square$ Infection & $15(23.4 \%)$ & $3(7.9 \%)$ & $\mathrm{p}=0.046$ \\
\hline$\square$ Prerenal failure & $6(9.4 \%)$ & $1(2.6 \%)$ & $\mathrm{p}=0.192$ \\
\hline$\square$ Malignant tumor & $6(9.4 \%)$ & 0 & $\mathrm{p}=0.051$ \\
\hline$\square$ After surgery & $3(4.7 \%)$ & $1(2.6 \%)$ & $\mathrm{p}=0.61$ \\
\hline$\square$ HHS/DKA & $2(3.1 \%)$ & 0 & $\mathrm{p}=0.27$ \\
\hline$\square$ AKA & $3(4.7 \%)$ & 0 & $\mathrm{p}=0.17$ \\
\hline$\square$ Others & $3(4.7 \%)$ & $2(5.3 \%)$ & $\mathrm{p}=0.89$ \\
\hline HHS, hyperosmolar hyperglycemic syndrome; DKA, diabetic ketoacidosis; AKA, alcoholic ketoacidosis \\
\hline
\end{tabular}

Table 3

Laboratory data

\begin{tabular}{|llll|}
\hline & AEM L(n= 64) & sRE-D $(\mathbf{n}=\mathbf{3 8})$ & P value \\
\hline WBC $(/ \mathrm{mL})$ & $12209 \pm 7616$ & $9323 \pm 4264$ & $\mathrm{p}=0.014$ \\
\hline Hemoglobin $(\mathrm{g} / \mathrm{dL})$ & $10.28 \pm 3.49$ & $9.46 \pm 2.93$ & $\mathrm{p}=0.21$ \\
BUN $(\mathrm{mg} / \mathrm{dL})$ & $52.02 \pm 42.73$ & $33.23 \pm 23.35$ & $\mathrm{p}=0.004$ \\
\hline Creatinine $(\mathrm{mg} / \mathrm{dL})$ & $2.45 \pm 3.39$ & $1.12 \pm 1.04$ & $\mathrm{p}=0.004$ \\
\hline Total protein $(\mathrm{g} / \mathrm{dL})$ & $5.77 \pm 1.61$ & $5.97 \pm 1.06$ & $\mathrm{p}=0.47$ \\
\hline Albumin $(\mathrm{g} / \mathrm{dL})$ & $3.00 \pm 1.02$ & $3.03 \pm 0.74$ & $\mathrm{p}=0.87$ \\
\hline Glucose $(\mathrm{mg} / \mathrm{dL})$ & $173 \pm 127$ & $136 \pm 52$ & $\mathrm{p}=0.048$ \\
\hline CRP $(\mathrm{mg} / \mathrm{dL})$ & $4.50 \pm 5.83$ & $1.53 \pm 2.30$ & $\mathrm{p}<0.001$ \\
\hline $\mathrm{pH}$ & $7.37 \pm 0.12$ & $7.43 \pm 0.05$ & $\mathrm{p}=0.004$ \\
\hline Lactate $(\mathrm{mmol} / \mathrm{L})$ & $4.98 \pm 4.36$ & $2.25 \pm 1.29$ & $\mathrm{p}<0.001$ \\
\hline
\end{tabular}

Value are presented as Mean \pm SD 
WBC, white blood cell count; BUN, blood urea nitrogen; CRP, C-reactive protein; AEML, acute esophageal mucosal lesion; sRE-D, severe reflux esophagitis, Los Angeles grade $D$

Endoscopic findings are shown in Table 4. Bleeding requiring endoscopic hemostasis $(p<0.05)$ and esophageal hiatal hernia $(p<0.001)$ were significantly more frequent in the sRE-D group, while gastric and duodenal ulcers $(p<0.05)$ were more frequent in the AEML group. There was no significant difference in atrophic gastritis $(p=0.47)$ between the two groups.

Table 4

Endoscopic features

\begin{tabular}{|llll|}
\hline & AEML & sRE-D & P value \\
\hline Endoscopic hemostasis & $5 / 64(7.8 \%)$ & $10 / 38(26.3 \%)$ & $\mathrm{p}=0.01$ \\
\hline Hiatal hernia & $46 / 62(74.2 \%)$ & $36 / 36(100 \%)$ & $\mathrm{p}<0.001$ \\
\hline Gastric ulcer & $14 / 64(21.9 \%)$ & $0 / 38(0 \%)$ & $\mathrm{p}=0.002$ \\
\hline Duodenal ulcer & $21 / 64(32.8 \%)$ & $2 / 38(5.3 \%)$ & $\mathrm{p}=0.001$ \\
\hline Gastric atrophy & $23 / 64(35.9 \%)$ & $11 / 38(28.9 \%)$ & $\mathrm{p}=0.47$ \\
\hline
\end{tabular}

AEML, acute esophageal mucosal lesion

SRE-D, severe reflux esophagitis, Los Angeles grade D

One patient in the AEML group had a stricture. In this case, vomiting and aspiration pneumonia were observed 1 month after the onset of symptoms. Upper endoscopy revealed an esophageal stricture (Figure 5). Although the nasal endoscope was successfully passed, balloon dilatation was difficult because of the stricture length $(10 \mathrm{~cm})$. Consequently, central venous feeding was instituted.

The shape of the proximal mucosal injury in the AEML group is shown in Table 5. The non-spiny nature of the proximal mucosal damage in the AEML group was classified as follows: the boundary of mucosal injury was not clear and changed gradually (Figure 6a) or the boundary of the injury was relatively clear (Figure 6b). However, most of the cases showed gradual changes (71.9\%).

Table 5

The shape of proximal mucosal injury in the acute esophageal mucosal legion group

\begin{tabular}{|ll|}
\hline Shape of proximal mucosal injury & $\mathbf{N}=64$ \\
\hline Boundary of mucosal injury changes in gradation & $46(71.9 \%)$ \\
\hline Boundary of the injury is relatively clear & $18(28.1 \%)$ \\
\hline
\end{tabular}


Therefore, AEML and SRE-D have distinctly different features, and it is reasonable to distinguish the two diseases by the shape of the proximal mucosal injury, even in our study, as in the study by Sakata et al [7].

In comparison with SRE-D, AEML was characterized by a younger age group, a higher prevalence of male patients, and comorbidities, such as infections, increased inflammatory response, worsening renal function, acidemia with increased lactate, and increased blood glucose levels. Endoscopic findings showed that hemostasis was not necessarily present in most cases, and that gastric and duodenal ulcers were common, with some cases of stenosis at a later date.

\section{Discussion}

This study aimed to verify whether it is reasonable to distinguish AEML and SRE-D by the characteristics of the proximal mucosal damage and to clarify the characteristics of AEML in comparison with SRE-D. Compared to the sRE-D group, the AEML group had more comorbidities, most of which were infections. The fact that many patients in the AEML group had been treated for malignancies was also considered to be a reason for their susceptibility to comorbid conditions, particularly infections. The significantly higher WBC and CRP levels in the AEML group than in the SRE-D group may have been due to the higher prevalence of coexisting diseases, such as infections. The possibility of AEML should be considered in patients with worsening systemic conditions, including infections.

The AEML group showed significantly higher lactate levels and acidemia than the SRE-D group. Lactate elevation and acidemia are caused by anaerobic metabolism and lactate production due to tissue hypoxia. The AEML group had more gastric and duodenal ulcers than the sRE-D group, suggesting that mucosal injury may have occurred because of impaired circulation, which may have been due to worsening of the patients' general condition because of coexisting disease. Additionally, the fact that the use of NSAIDs was significantly higher in the AEML group supports this hypothesis, as the use of NSAIDs may contribute to impaired circulation [11].

The BUN and creatinine levels were also significantly higher in the AEML group. Compared with those in the SRE-D group, patients in the AEML group were more likely to exhibit renal dysfunction and volume loss due to deterioration of their general condition. In particular, volume loss resulted in a tendency for the development of microvascular insufficiency.

The blood glucose levels were significantly higher in the AEML group. This is because patients with AEML are under physical and psychological stress, as well as because some patients had coexisting diabetic ketoacidosis (DKA) and hyperosmolar hyperglycemic syndrome (HHS). In particular, DKA and HHS are diseases that cause severe circulatory insufficiency, which may lead to esophageal mucosal damage and the development of AEML.

The possibility of AEML been caused by gastric acid reflux has been reported in previous studies [1-4]. However, in the present study, the use of PPI was higher in the AEML group $(<45 \%)$, and esophageal hiatal 
hernia was less common in the sRE-D group. Since atrophic gastritis was also comparable, it can be considered that there was no significant difference in gastric acid secretion capacity between the two groups. Compared to the SRE-D group, AEML development seemed to be more related to inadequate peripheral circulation than to gastric acid secretion.

The frequency of endoscopic hemostasis in the AEML group was $7.8 \%$, which was significantly lower than that in the SRE-D group. Bleeding was less frequent in the AEML group, but this did not mean that endoscopic procedures were unnecessary. Patients with AEML improve quickly with conservative treatment $[1,2,4,7]$. If there is no bleeding on upper endoscopy, it is important to improve the general condition of the patient, including the treatment of comorbidities.

In the AEML group, a case of stenosis was observed after healing. Thus, the incidence of stenosis was only $1.6 \%$ in our study, whereas previous reports have shown an incidence of $4 \%$ [4]. The most common timing for the stenosis is at 7-10 days after onset [5], but it can also occur within 1 month, as in this case. Therefore, it is necessary to perform follow-up at an appropriate time, considering the risk of stenosis.

In this study, we differentiated SRE-D from AEML by the presence of spiculation on the proximal edge of the esophageal mucosa, as reported by Sakata et al. [7]. The characteristics of proximal mucosal damage in AEML were classified into two types as shown in Figure 6, but many of them changed gradually. The reason for this is that the proximal mucosal damage in SRE is clearly defined, and the involvement of gastric acid reflux may be strong in the clear boundary. However, this has not been proved yet. The squamocolumnar junction has a clear border, but the proximal mucosal damage is gradual, which is typical of AEML.

The reason for the significantly higher number of older women in the SRE-D group was considered to be the effect of hunchback. Older women are more prone to osteoporosis and hunchback than men. Chronic acid reflux and decreased clearance due to hunchback may predispose older women to SRE-D.

The novel findings of this study are as follows: 1 ) the distinction between SRE-D and AEML, which is more relevant in clinical practice; 2) suggesting that microcirculatory disturbances may be more relevant to the development of AEML than acid reflux, compared with SRE-D; 3) two types of erosions can be distinguished in AEML; and 4) the possibility of stenosis in AEML.

This was a retrospective, single-center study, which may limit the generalizability of the results. It should also be noted that the statistically significant difference in AEML in this study is only a result of comparison with SRE-D and may not be a significant feature of AEML itself.

\section{Conclusions}

AEML and SRE-D have distinctly different features, and it is reasonable to distinguish the two diseases by the shape of the proximal mucosal injury. AEML, unlike SRE-D, may be caused by microcirculatory 
disturbances related to comorbidities rather than gastric acid reflux. AEML is still not widely recognized. In patients with worsening general condition, when upper gastrointestinal bleeding occurs, it is important to consider this disease and to improve the general condition, and to be prepared for the possibility of stenosis.

\section{Abbreviations}

AEML: acute esophageal mucosal lesions; sRE: severe reflux esophagitis; sRE-D: severe reflux esophagitis, Los Angeles grade D; NSAIDs: nonsteroidal anti-inflammatory drugs; WBC: white blood cell; CRP: C-reactive protein; BUN: blood urea nitrogen; PPI: proton-pump inhibitors: DKA: diabetic ketoacidosis; HHS: hyperosmolar hyperglycemic syndrome

\section{Declarations}

\section{Ethics approval and consent to participate}

All procedures were performed in accordance with the ethical standards established in the 1964 Declaration of Helsinki and its later amendments. The study was reviewed and approved by the institutional review board of the Future Medical Research Center Ethical Committee (IRB no. TGE01484024). Owing to the retrospective study design, informed consent was obtained from all participants by the opt-out method on our hospital website and in-hospital posting.

\section{Consent for publication}

Not applicable

\section{Availability of data and materials}

All data analyzed in this study are available from the corresponding author on reasonable request.

\section{Completing interests}

The authors declare that they have no competing interests.

\section{Funding}

This research did not receive any specific grant from funding agencies in the public, commercial, or notfor-profit sectors.

\section{Authors' contributions}

All authors have contributed to and approved the final manuscript. Cl collected data, made analysis and wrote the manuscript; AS revised the manuscript; SM, KK2, MK supervised this research; CS, TN, JK, KK1, $\mathrm{KS}, \mathrm{MM}$ contributed equally to this work. 
Acknowledgments

We would like to thank Editage (www.editage.com) for English language editing.

\section{References}

1. Tsumura T, Maruo T, Tsuji T, Osaki Y, Tomono N. TWELVE CASES OF ACUTE ESOPHAGEAL MUCOSAL LESION. Dig Endosc. 2006;18:199-205.

2. Tatsumi R, Ohta T, Matsubara Y, Yoshizaki K, Sakamoto J, Sato R, et al. Examination of the survey of subtypes of acute esophageal mucosal lesion. Gastroenterol Endosc. 2014;56:3775-85.

3. Ihara Y, Hizawa K, Fujita K, Matsuno Y, Sakuma T, Esaki M, et al. Clinical features and pathophysiology of acute esophageal mucosal lesion. Nihon Shokakibyo Gakkai Zasshi. 2016;113:642-46.

4. Kawauchi H, Ohta T, Matsubara Y, Yoshizaki K, Sakamoto J, Amitsuka H, et al. Clinicopathological study of acute esophageal mucosal lesion. Nihon Shokakibyo Gakkai Zasshi. 2013;110:1249-57.

5. Gurvits GE, Shapsis A, Lau N, Gualtieri N, Robilotti JG. Acute esophageal necrosis: A rare syndrome. J Gastroenterol. 2007;42:29-38.

6. DiBaise JK. The LA classification for esophagitis: A call for standardization. Am J Gastroenterol. 1999;94:3403-4.

7. Sakata Y, Tsuruoka N, Shimoda R, Yamamoto K, Akutagawa T, Sakata N, et al. Comparison of clinical characteristics of patients with acute esophageal mucosal lesion and those with severe reflux esophagitis. Digestion. 2019;99:275-82.

8. Kimura K, Takemoto T. An Endoscopic Recognition of the Atrophic Border and its Significance in Chronic Gastritis. Endoscopy. 1969;1:87-97.

9. Redéen S, Petersson F, Jönsson KA, Borch K. Relationship of gastroscopic features to histological findings in gastritis and Helicobacter pylori infection in a general population sample. Endoscopy. 2003;35:946-50.

10. Kanda Y. Investigation of the freely available easy-to-use software 'EZR' for medical statistics. Bone Marrow Transplant. 2013;48:452-8.

11. Wallace JL, Reuter B, Cicala C, Mcknight W, Grisham MB, Cirino G. Novel nonsteroidal antiinflammatory drug derivatives with markedly reduced ulcerogenic properties in the rat. Gastroenterology. 1994;107:173-9.

\section{Figures}




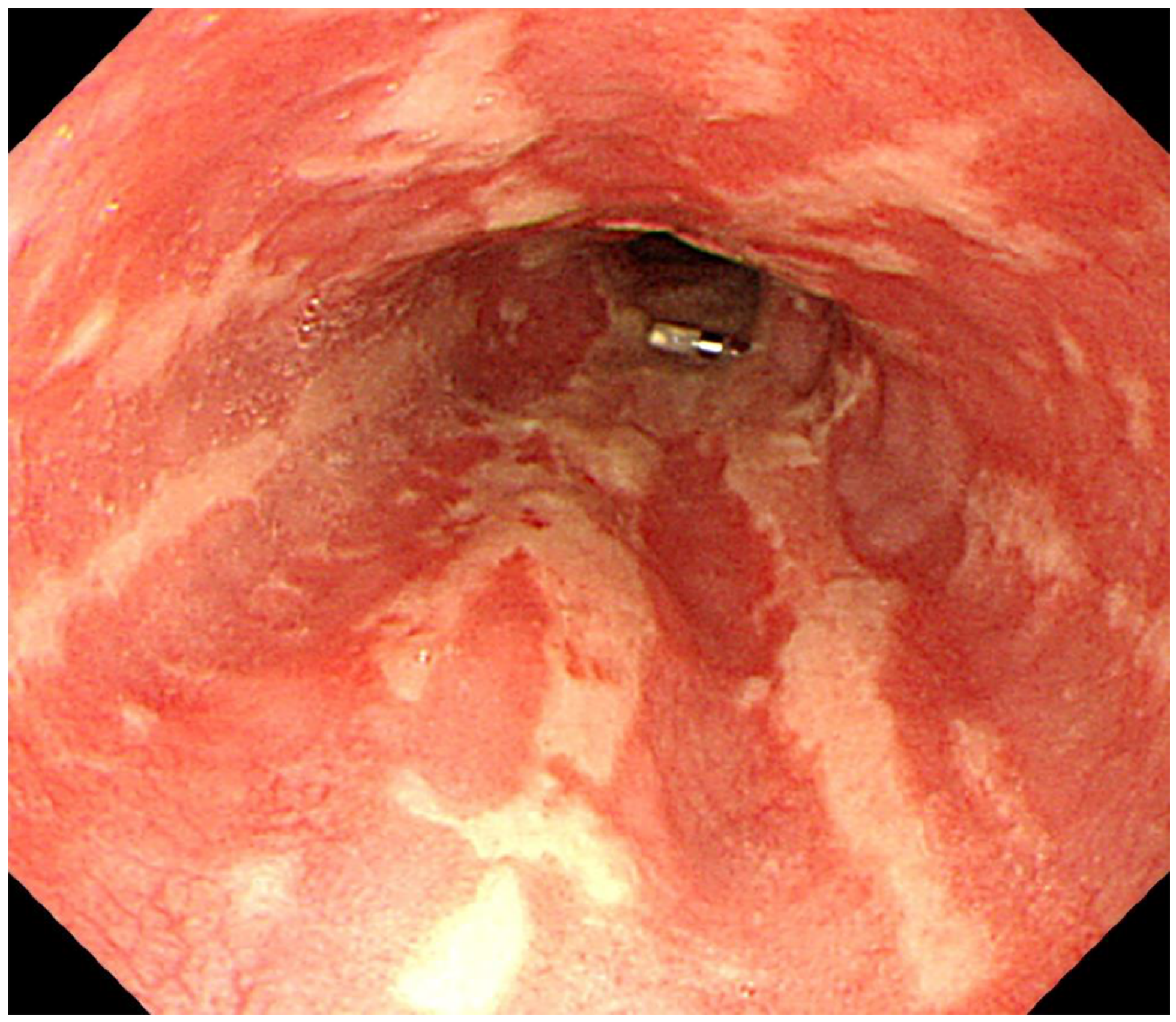

\section{Figure 1}

Endoscopic features of severe reflux esophagitis, Los Angeles grade D.

Fused mucosal lesions are observed and spiny pointed erosions are observed on the proximal edge of the side. 

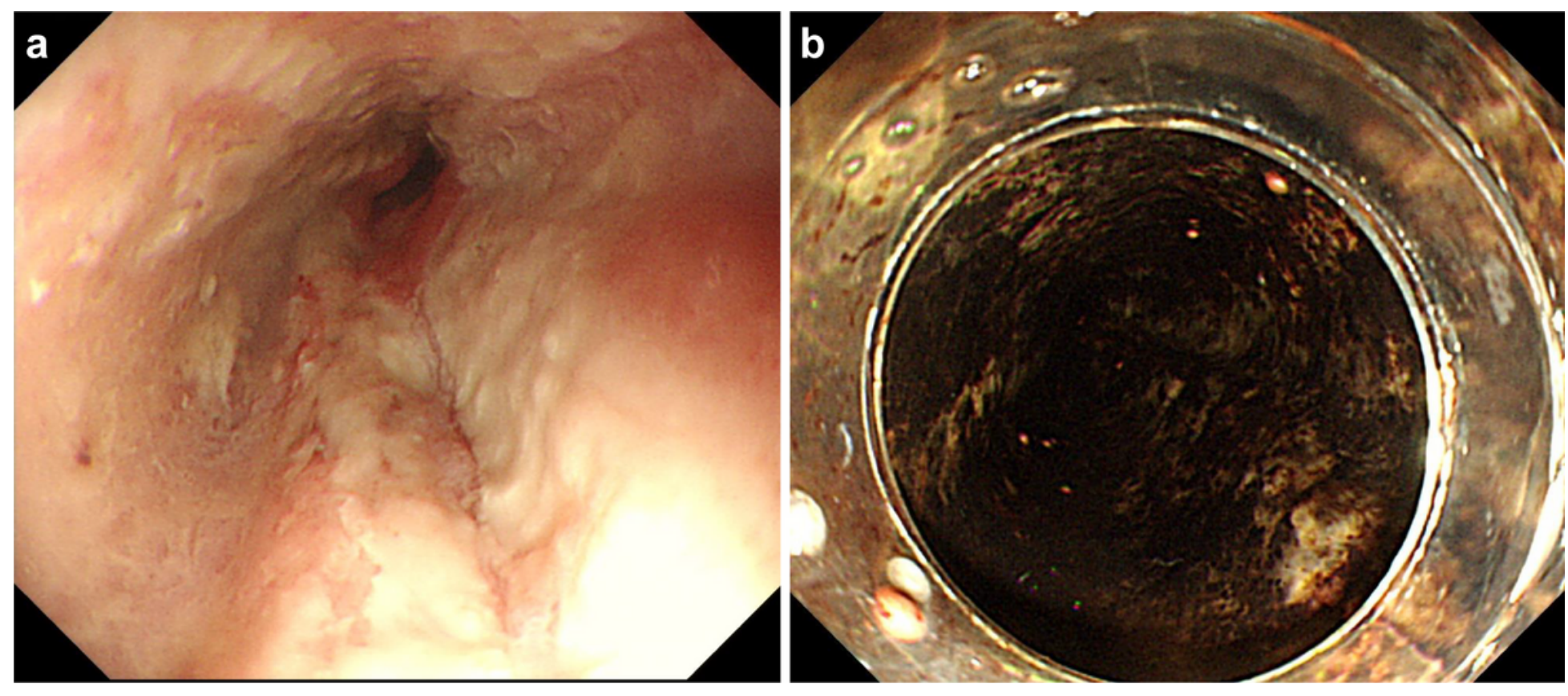

Figure 2

Endoscopic features of acute esophageal mucosal lesions.

Circumferential diffuse mucosal injury involves at least the entire lower third of the esophagus, and includes black and non-black esophagitis. The proximal mucosa is not spiculated.

a: Non-black esophagus

b: Black esophagus
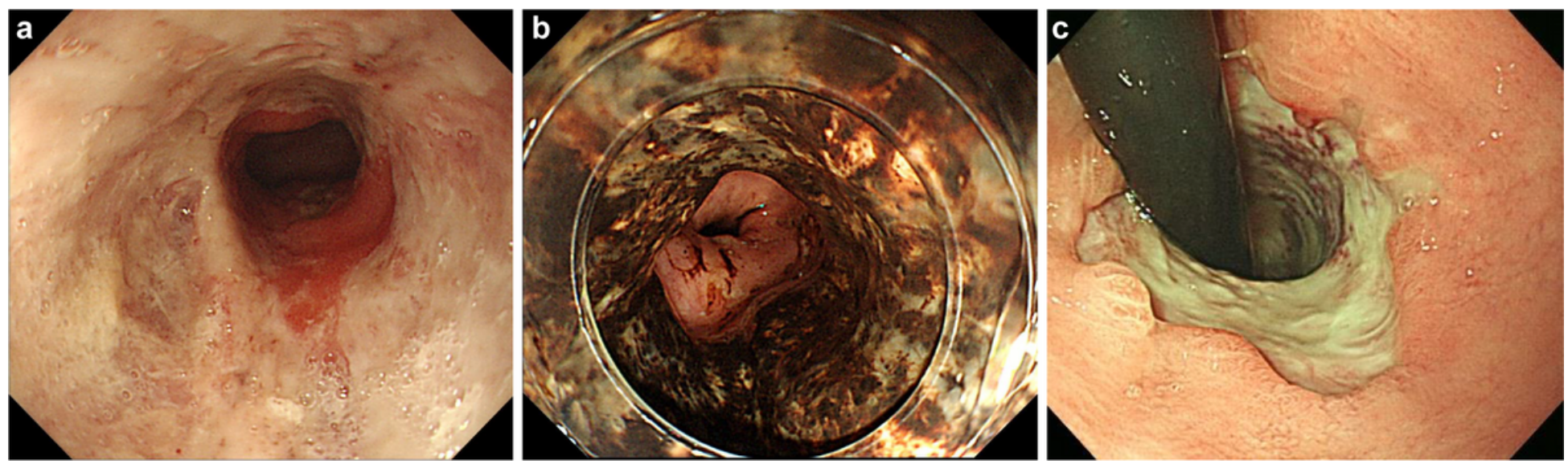

Figure 3

Endoscopic features of acute esophageal mucosal lesions (squamocolumnar junction), including black and non-black esophagitis.

The inflammation of the mucosa is sharply bordered by the squamocolumnar junction 
a: Non-black esophagus

b: Black esophagus

c: Retroflex view of the squamocolumnar junction

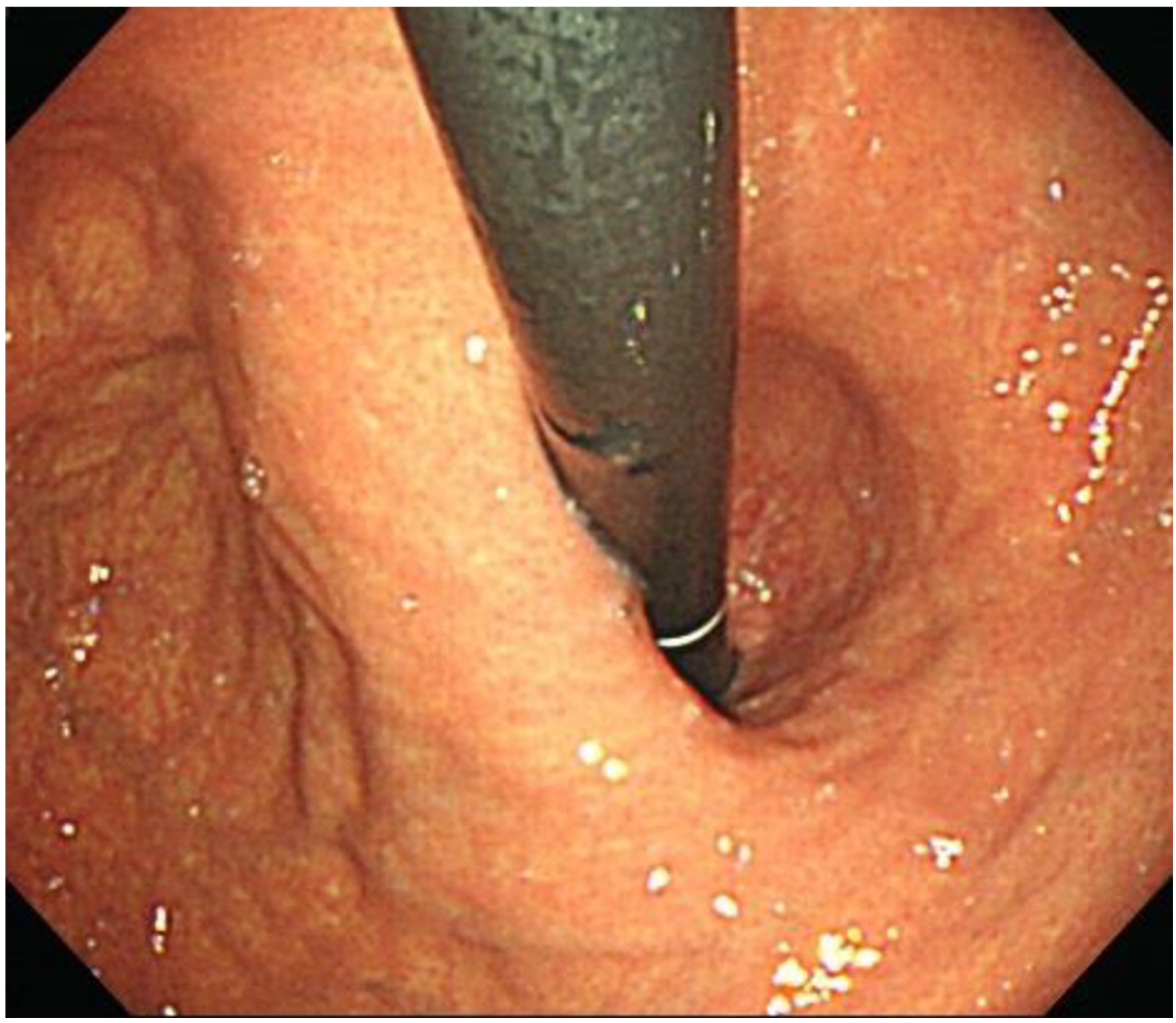

Figure 4

Endoscopic findings of esophageal hiatal hernia.

When the gap between the endoscope scope and hilum is observed during the retroflex view, the hilum is considered open. This was used for indirect evaluation of the presence of hiatal hernia. 


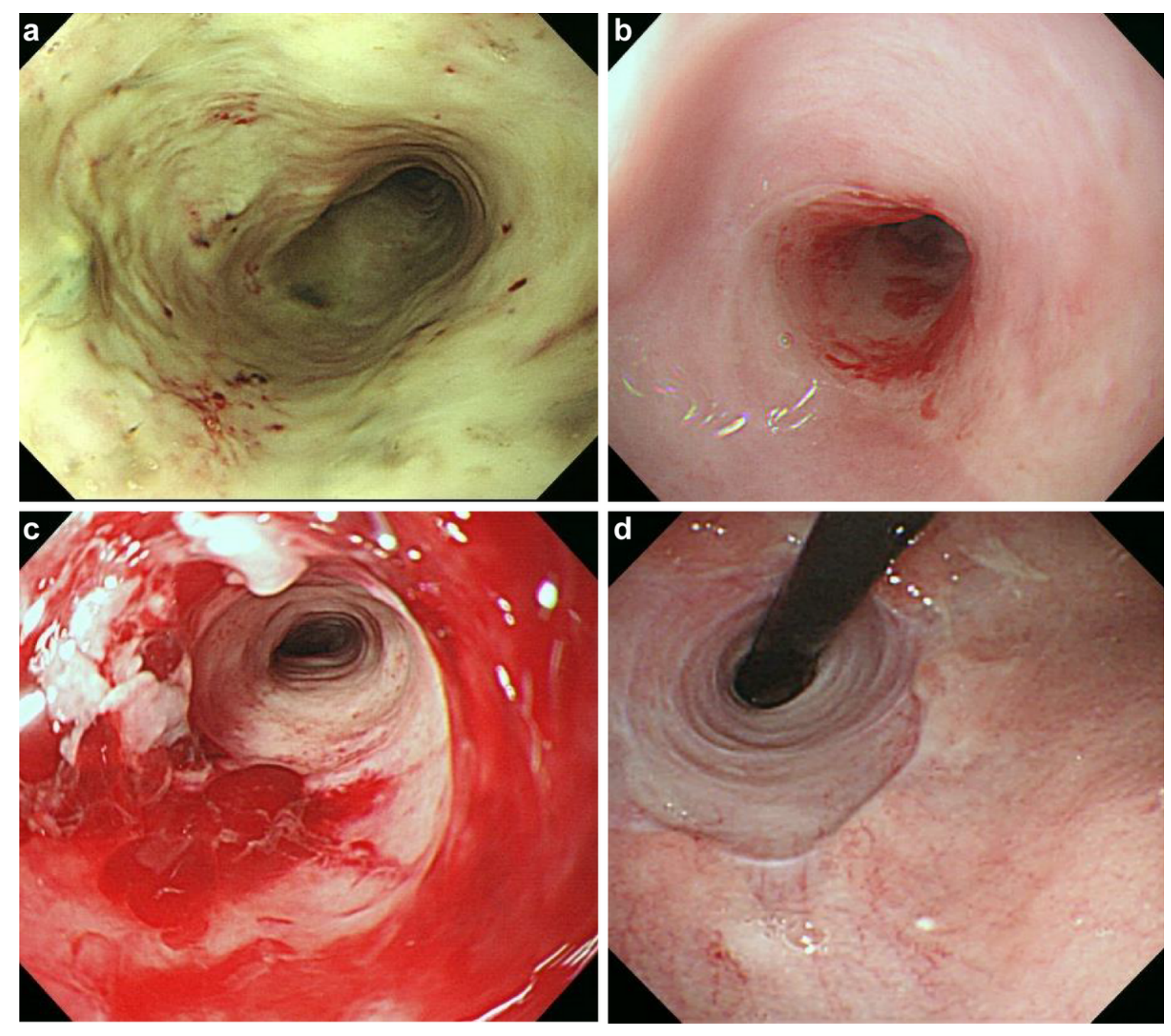

Figure 5

Endoscopic findings in a stenosis case.

a: Endoscopic image at the time of onset. Generalized esophageal ulcer is observed.

b, c, d: Endoscopic image after formation of stenosis.

b, c: The stenosis is too severe to allow passage of the oral endoscope.

d: Retroflex view of esophagogastric junction. The nasal endoscope managed to pass through the stenosed region. 


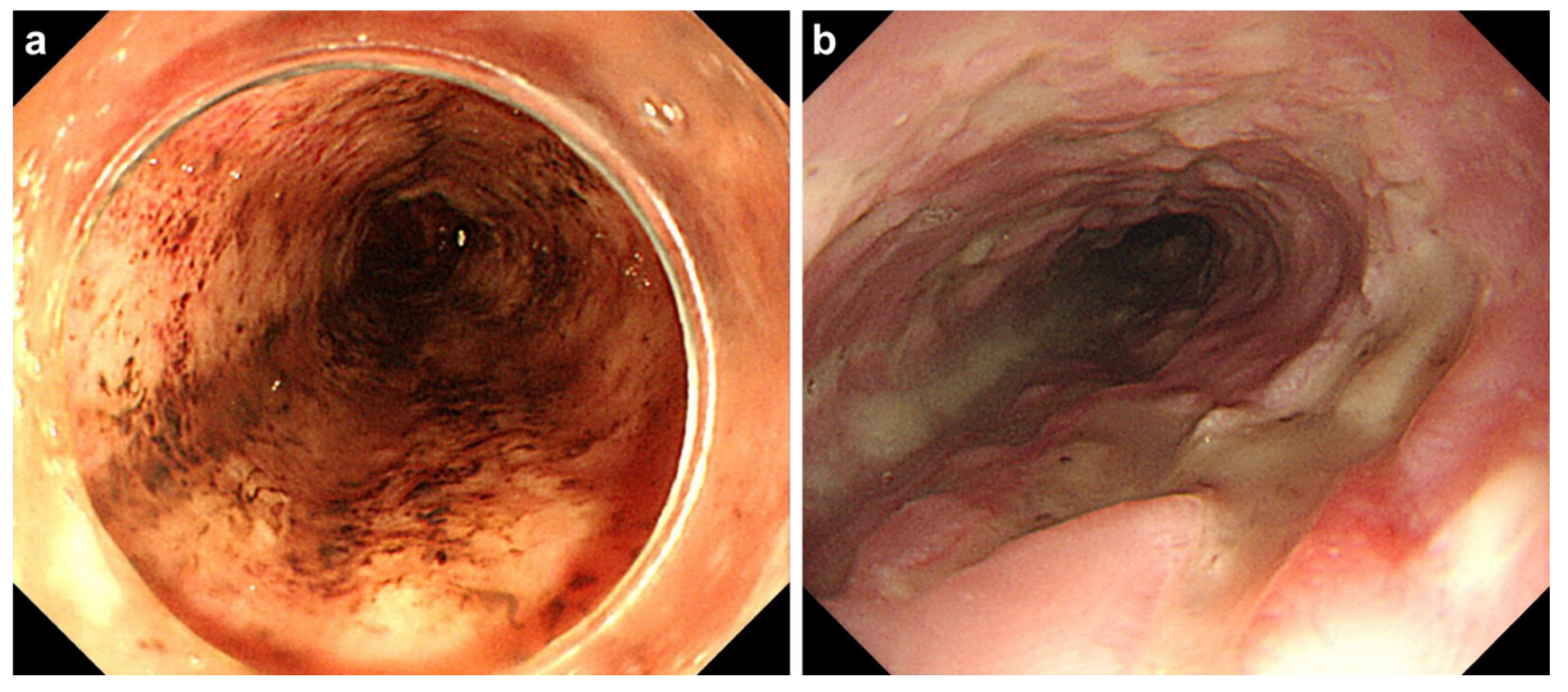

Figure 6

Shape of proximal mucosal injury in the acute esophageal mucosal lesion group.

a: The boundary of mucosal injury is not clear and gradual changes are observed.

b: The boundary of mucosal injury is relatively clear. 